\title{
Urbaner Lebensraum
}

(Ein systemtheoretischer Ansatz zu aktualgeographischer Forschung)

\section{Einleitung}

Geographische Stadtforschung sollte sich unseres Erachtens vermehrt mit aktuellen Problemen urbaner Lebensräume auseinandersetzen, wie sie etwa MITSCHERLICH $(1972,9)$ aufgezeigt hat: «Die hochgradig integrierte alte Stadt hat sich funktionell entmischt. Die Unwirtlichkeit, die sich über diesen neuen Stadtregionen ausbreitet, ist niederdrückend. Die Frage lautet: $\mathrm{Mu} ß$ das so sein, ist das unausweichlich?»

In einer Gemeinschaftsdissertation versuchen wir, den Prozeß der «funktionellen Entmischung», wie ihn MITSCHERLICH nennt, und seine Ursachen zu erfassen. Diese Untersuchungen sollten es ermöglichen, die heutige Stadt in ihrer Veränderung besser zu verstehen. Somit könnte auch der Bevölkerung aufgezeigt werden, wo die Ursachen ihres weitverbreiteten Unbehagens liegen und welche Lösungswege beschritten werden müßten. Der Arbeitstitel der Dissertation lautet: Basel als urbaner Lebensraum. Prozesse und Dynamik eines urbanen Systems, aufgezeigt an Kleinbasler Arbeitervierteln und am suburbanen Profilband BirsfeldenRheinfelden.

Der heutige Wissensstand läßt eine theoretische Durchdringung des stadtgeographischen Raumes zu. Wir stellen deshalb der empirischen Arbeit ein Stadtmodell voran. Dieses wurde bisher in Diskussionen und während der letzten vier Jahre durch empirische Kleinuntersuchungen auf seine Plausibilität hin überprüft. Wir stellen das Modell mit dieser Veröffentlichung einem weiteren Kreise zur Diskussion, während wir es gleichzeitig empirisch nachprüfen. Da das Modell als System aufgefaßt wird, ist es notwendig, allgemeine Bemerkungen zur Systemtheorie vorauszuschicken.

Im Artikel wird demnach mit den Kapiteln «Systemtheorie» und "Auseinandersetzung mit bisherigen Konzepten» ein systemtheoretischer und thematischer Rahmen entworfen, in welchem unser Modell erst verständlich wird.

\section{Systemtheorie}

Um Löșungen für Probleme der Stadt zu entwerfen, müssen wir von der hochkomplexen Realität abstrahieren. Mit der Systemtheorie verfügen wir über ein präzises Begriffsinstrumentarium, das erlaubt, für die Problemlösung Wesentliches von Unwesentlichem zu trennen. Die Systemtheorie eignet sich zur Darstellung des Erkenntnisvorganges und zur Überprüfung seiner inneren Logik.

\section{Definitionen zur Systemtheorie}

Um Mißverständnissen vorzubeugen, werden vorerst häufig verwendete Begriffe definiert:

1. System: «Menge von Elementen und Menge von Relationen, die zwischen diesen Elementen bestehen» (KLAUS 1969, 634). Vorläufig fassen wir das System als abstraktes Gebilde auf, das wir erst weiter unten für unseren Gebrauch konkretisieren werden (vgl. HARBORDT 1974, 49 f.).

2. Elemente: Grundlegende Bestandteile eines Systems, die in bezug auf dieses System als nicht weiter zerlegte Einheiten aufgefaßt werden. Dabei müssen sich die Elemente gegenseitig ausschließen (FRIEDRICHS 1973, 93 und Lexikon zur Soziologie 1974, 158).

3. Relationen: Beziehungen oder Verbindungen zwischen Elementen. Die Art dieser Beziehungen (logische, distanzielle usw.) wird durch das System festgelegt.

4. Struktur: «... das Beziehungsmuster der Elemente, das durch die Menge der Relationen und durch die Art ihrer Anordnung gekennzeichnet ist) (HARBORDT 1974, 45).

5. Verhalten: «... die von Einwirkungen der Umwelt bedingten Reaktionen, bzw. Rückwirkungen des Systems auf seine Umwelt» (HARBORDT 1974, 47).

6. Umwelt: Alle jene Systeme, die mit mindestens einem Element zu mindestens einem Element des Ausgangssystems in Beziehung stehen. «Prinzipiell ist die Umwelt eines Systems differenzierbar nach Systemen gleicher oder verschiedener Qualität, verglichen mit dem Ausgangssystem» (Lexikon zur Soziologie 1973, 707). «Für ein bestimmtes Element stellen alle anderen Elemente des Systems die Umwelt dan》 (HARBORDT 1974, 45).

Werner Laschinger und Lienhard Lötscher, Geographisches Institut der Universität Basel, Klingelbergstraße 16, 4056 Basel 


\section{Erkenntnisvorgang}

Im folgenden wird der Erkenntnisvorgang mit Hilfe der Systemtheorie beschrieben. Da sie eine Reduktion zuläßt ohne zu simplifizieren, ist sie dazu sehr geeignet. In Abb. la ist der Erkenntnisvorgang schematisch dargestellt.

Die totale Realität (in ihrer unendlichen räumlichen und zeitlichen Ausprägung und mit ihren unendlichen maßstäblichen Aspekten) ist als solche nicht faßbar. Ein umfassend isomorphes Abbild der totalen Realität könnte nur wieder sie selbst sein. Jeder Erkenntnisvorgang ist aus diesem Grund damit beschäftigt, von dieser Totalität Abstriche vorzunehmen, d.h. die Komplexität zu reduzieren. Für die Alltagserfahrung genügt eine auf impliziten Kriterien beruhende Auswahl. Die Wissenschaft hingegen bemüht sich darum, explizite Auswahlkriterien zu erarbeiten.

Das Kontinuum der totalen Realität wird in einem ersten Schritt in den drei Dimensionen Zeit, Raum und Maßstab durch die Zerlegungsebene unterbrochen. Als erster, aber noch allgemeiner Reduktionsschritt ist sie Voraussetzung der Modellbildung. Die Zerlegungsebene wird durch Forschungstradition und Stand der Theoriebildung festgelegt und muß nur mittelbar auf die Problemlösung bezogen sein. Dieser Bezug ist hingegen durch die weiteren Reduktionsschritte immer unmittelbarer herzustellen.

Der so entstandene Ausschnitt wird als reales System definiert, zumal schon von der totalen Realität grundsätzlich angenommen wird, sie könne als System aufgefaßt werden. Das reale System steht in der Isomorphierelation (umkehrbar eindeutige Zuordnung aller Elemente und Relationen) zur totalen Realität.

In einem zweiten Schritt wird aufgrund vorwissenschaftlicher und wissenschaftlicher Kenntnisse ein Modell entworfen, das als System dargestellt wird und dem realen System homomorph sein soll. Dadurch werden gewisse Beziehungen und Elemente relevant gesetzt.

Die Elemente und vor allem ihre gegenseitigen $\mathrm{Ab}$ hängigkeiten oder Beziehungen werden exakt bestimmt, weil wir das Modell als System definieren. Dies ermöglicht eine übersichtliche Darstellung des Modells und gleichzeitig die Kontrolle seiner inneren Logik.

Die dem Modell entsprechenden Elemente und Beziehungen der Realität nennen wir empirisches Relativ (vgl. KRIZ 1973, 26 ff.). Davon werden Daten erhoben, welche das numerische Relativ bilden. Danach wird durch den Rückbezug auf das empirische Relativ der Aussagewert des numerischen Relativs interpretiert. Dadurch sind Rückschlüsse auf das Modell und vom Modell auf das reale System möglich.

Abb. 1b stellt das empirische Relativ und das reale System als Teilmenge der totalen Realität dar, wobei durch das Rechteck des realen Systems auf die Isomorphie-, durch die Kreise des empirischen Relativs auf die Homomorphierelation hingewiesen werden soll.

\section{Geographische Systeme}

\section{Aktualgeographisches System}

Es gilt nun, die systemtheoretischen Überlegungen auf eine allgemeingeographische und auf unsere konkrete Fragestellung $\mathrm{zu}$ übertragen. Entsprechend wird die Zerlegungsebene festgelegt. Dabei wird als reales System das aktualgeographische System abgegrenzt. (Zur Bedeutung aktualgeographischer Analysen vgl. GAL LUSSER 1970, 15; 1971, 175.) Die Dimensionen erhalten folgende Ausprägungen:

1. Zeit: Aus dem zeitlichen Kontinuum wird nur die Gegenwart berücksichtigt. Es soll sich dabei um einen aktuellen Ausschnitt handeln.

2. Raum: Der räumliche Ausschnitt umfaßt die Oberfläche der Erde mit all ihren natürlichen und durch die Gesellschaft geschaffenen Ausprägungen wie sie im geographischen i-, j-, k-Koordinatennetz festgehalten werden können.

3. Maßstab: Es wird ein mittlerer Bereich ausgeschieden, der etwa von $4,01 \times 10^{9} \mathrm{~cm}$ (Erdumfang) bis $10^{3} \mathrm{~cm}$ reicht (vgl. HAGGET 1965, 5).

\section{Urbanes System}

Die weitere Reduktion erfolgt durch das Modell, das aus verschiedenen, hierarchisch geordneten Systemen aufgebaut wird. Ein aktualgeographisches System müßte neben einem urbanen und einem ruralen auch physische Systeme aufweisen. Dies dürte fachtheoretisch interessante Perspektiven eröffnen (vgl. auch: Autorengemeinschaft 1973). Aus Platzgründen beschränken wir uns aber - unserer Fragestellung entsprechend - ein Modell für den «aktualgeographischen 
urbanen Raum» zu entwerfen. Wir nennen dieses Modell, zumal es als System betrachtet wird, urbanes System (vgl. Abb. 2).

Exkurs: primäre und sekundäre Aufgabe des Raumes Im urbanen System bilden die untergeordneten Produktions- und Konsumtions-Systeme die Elemente. Die Aufteilung in diese beiden Systeme beruht auf der Annahme, daß dem Raum grundsätzlich eine primäre und eine sekundäre Aufgabe zugeschrieben werden kann.

Die Aufgabe, Grundlage für die Herstellung von Gütern (im weitesten Sinne) zu sein, die gesamtgesellschaftlich von Bedeutung sind, nennen wir die primäre Aufgabe des Raumes. Es ist nur wichtig, daß sie in einem bestimmten Raum (und nicht in einem anderen) produziert werden. Dieser Raum kann auch als Wirtschaftsraum bezeichnet werden.

Die sekundäre Aufgabe des Raumes besteht darin, Lebensraum für die Bevölkerung zu sein, die die oben erwähnten Güter herstellt. Dabei kommt es nicht in erster Linie auf die Art der Güter an, sondern nur darauf, $d a \beta$ Güter hergestellt werden und daß räumliche Einrichtungen vorhanden sind, die das tägliche Leben ermöglichen. Es ist wichtig, wie diese Einrichtungen verteilt sind.

Mit den Begriffen Wirtschaftsraum und Lebensraum nehmen wir in etwas abgeänderter Form Bezug auf das «basic/non basic function» Konzept (vgl. MONKHOUSE 1973, 34).

\section{Elemente des urbanen Systems}

Dementsprechend werden für das urbane System diese beiden Aspekte als Elemente ausgeschieden. Das System Wirtschaftsraum entspricht den primären Funktionen, das System Lebensraum den sekundären. Wir legen das Hauptgewicht unserer Modellbildung auf das System Lebensraum, weil die Probleme, die wir zu lösen versuchen, diesen Bereich betreffen.

Das System Wirtschaftsraum ziehen wir nur bei, wenn dies zur Erklärung von Prozessen im System Lebensraum notwendig ist. Dies trifft für den Urbanisierungsprozeß, als wichtigsten Prozeß im System Wirtschaftsraum, zu. Die Ursachen des Urbanisierungsprozesses könnten durch ein DYN-System Wirtschaftsraum erfaßt werden. Die beiden DYN-Systeme sind aus den Kriterien aufgebaut, die die Prozesse im Wirtschaftsraum-oder Lebensraum-System bewirken.

\section{Das System Lebensraum}

Das System Lebensraum ist auf den Daseinsgrundfunktionen aufgebaut. Diese Grundfunktionen sind umstritten; einmal wegen des kategorialen Rahmens der «Daseinsbedürfnisse» des Menschen und weiter wegen der Nähe zum Funktionalismus. Beide Ansätze zur Kritik und zusätzlich die Grundvorstellung zur Raumkonzeption werden anhand einiger wichtiger Konzeption diskutiert. Diese Begriffsklärung ist auch deshalb nötig, um allfällige Vorurteile gegenüber unseren zentralen Begriffen abzubauen.

\section{Auseinandersetzung mit bisherigen Konzepten}

Ansätze der angewandten Forschung, die sich mit Stadt und Stadtplanung auseinandersetzen, geben in der Regel vor:

1. Man brauche nur Ideen zu haben, dann könne man planen; d.h. um Mißstände zu beheben, benötige man lediglich «genialische Einfälle». Diese könnten dann durch die Organe der Raumplanung ohne weiteres in die Realität umgesetzt werden.

2. Wirtschaftliche Entwicklungen und Mechanismen sowie Machtverhältnisse könnten vernachlässigt oder ausgeklammert werden.

Diesen Grundannahmen muß entgegengetreten werden; denn Planungsideen sind von vorneherein zum Scheitern verurteilt, wenn wirtschaftspolitische Gesichtspunkte nicht berücksichtigt werden. Gerade die wichtigsten Standortentscheidungen werden «...von denjenigen getroffen, die die Verfügungsgewalt über die Produktionsmittel (bzw. die Investitionsmittel) besitzen» (LENG 1973, 129).

Diese grundsätzlichen Anmerkungen gilt es im Auge zu behalten, wenn wir im folgenden drei Forschungskonzeptionen herausgreifen, die für unseren Ansatz von Bedeutung sind: Das funktionale Konzept der "Charta von Athen", die Konzeption der Grunddaseinsfunktionen wie sie PARTZSCH entwickelte und, auf beiden aufbauend, der «Münchnen» sozialgeographische Ansatz.

\section{Das funktionale Konzept der "Charta von Athen»}

Die funktionale Methode wurde bereits 1927 durch BOBEK in die Geographie eingeführt. Ihre große Bedeutung für die Stadtgeographie erlangte sie aber erst auf dem Umweg über Städtebau und Stadtplanung. 
Dort fand das funktionale Prinzip vor allem Zugang durch die Thesen der "Charta von Athen» (LE CORBUSIER, 1943).

Die Grundgedanken der dreiteiligen Charta wurden in den Jahren 1928-1933 von einer Gruppe international anerkannter Architekten entwickelt, die sich zum CIAM (Congrès Internationaux d'Architecture Moderne) zusammengeschlossen hatten.

Im dritten Teil, der Leitsätze für die Planung und deren Verwirklichung enthält, vertraten die Kongreßteilnehmer die Ansicht, die städtebaulichen Mißstände lösen oder zumindest das «Funktionieren» der Städte sicherstellen zu können, indem sie vier Schlüsselfunktionen: Wohnen, Arbeiten, Sich-Erholen (Freizeit), Sich-Bewegen (Verkehr), klar getrennte Bereiche zuordneten (Sätze 77 und 78 der Charta). Jeder Funktionsbereich sollte ein ganzes Quartier umfassen.

Mit solchen monofunktionalen Vierteln glaubten die Architekten, die Städte gliedern und ordnen, also überschaubar machen zu können. "Aus der berechtigten Kritik einer wahl- und systemlosen Funktionsmischung, wie sie sich in großem Umfang in alten Mischgebieten zeigte», schreibt WIEGAND $(1973,18)$, «verfolgte der funktionelle Städtebau nun das andere Extrem: die undifferenzierte Trennung der Funktion Wohnen und Arbeiten").

Der funktionelle Städtebau hätte sich wohl kaum durchzusetzen vermocht und wäre nicht so stark in der heutigen Planungs- und Baugesetzgebung verankert, wenn sich seine Ideen nicht mit wirtschaftlichen Interessen Je' ${ }^{\prime}$ t hätten.

\section{Das Konzept der Grunddaseinsfunktionen}

Der Begriff der Grunddaseinsfunktionen wurde von PARTZSCH (1964), wie LENG $(1973,122$ f.) bemerkt «. . in Anlehnung an die ,Charta von Athen' sowie an isBARYs Konzeption der ,Funktionsgesellschaft' (1971/ 3-5)», eingeführt. PARTZSCH vertritt die Ansicht, daß wir heute in einer Gesellschaftsepoche leben, deren Raumansprüche durch eine Skala von Funktionsmerkmalen gekennzeichnet ist, «... die sich als kategoriale Grundfunktionen des Menschen in der Gesellschaft erweisen» $(1966,518)$. Ziel dieser Funktionsgesellschaft sei, die Grunddaseinsfunktionen «... - arbeiten, wohnen, sich versorgen, sich bilden, sich erholen, am Verkehr teilnehmen und in Gemeinschaft sein - wieder in ein ausgewogenes, den legitimen Erwartungen und Ansprüchen der Gesellschaft entsprechendes Verhält- nis zueinander zu bringen, d.h. sie optimal funktionieren zu lassen» $(1966,517)$.

PARTZSCH vertritt damit wie die Teilnehmer am CIAM die Auffassung, jeder Funktion könne ein räumlich getrennter Bereich zugeordnet werden. Diese Bereiche müßten dann aneinandergefügt und die dazwischenliegenden Distanzen mit Nahverkehrsmitteln überbrückt werden.

Überschreiten aber die Funktionsbereiche und Distanzen eine gewisse Größe, so nehmen die Schwierigkeiten sprunghaft zu: Einerseits werden die monofunktionalen Gebiete nur während weniger Tages- oder Nachtstunden genutzt, so daß sie während der übrigen Zeit veröden. Andererseits entstehen immer größere Verkehrsprobleme, da sich der tägliche Transport der Nutzer über immer größere Distanzen und während relativ kurzer Zeit abwickelt. Volkswirtschaftlich gesehen bewirkt das Konzept der Funktionstrennung also auch ein Ansteigen der sozialen Kosten.

Warum haben aber die Ideen des Funktionalismus, die zwar einige Verbesserungen gebracht haben, zum "geplanten Chaos» geführt? Die Hauptursache dürfte darin zu suchen sein, daß menschliches Leben sich nicht - wie der Funktionalismus meint - funktional aufteilen läßt. Menschliche Bedürfnisse lassen sich eben nur vermeintlich systematisieren. Deshalb können von den Bedürfnissen her weder Funktionen noch dazugehörige Bauten abgeleitet werden (vgl. dazu die Kritik von BURCKHARDT und FÖRDERER 1973, am «Bauen nach Themen»).

\section{Der "Münchner» sozialgeographische Ansatz}

Auch der «Münchnen» sozialgeographische Ansatz krankt etwas an diesen Ideen. Nicht umsonst wurde er von BIRKENHAUER $(1974,501)$ als «geographischer Funktionalismus» apostrophiert.

Die «Konzeption der Sozialgeographie», wie sie RUPPERT und SCHAFFER (1969) vorstellten, hat zumindest auf die deutschsprachige Humangeographie sehr anregend gewirkt. Dabei handelt es sich offensichtlich um «... eine Zusammenfassung der theoretischen und methodischen Aussagen der Münchner Schule der Sozialgeographie (HARTKE und SCHÜLER) sowie derjenigen BOBEKs» (LENG 1973, 121). RUPPERT und SCHAFFER wollen ihre Konzeption $(1969,121)$ als «... Weiterentwicklung der funktionalen Anthropogeographie unter sozialwissenschaftlichen Aspekten» verstanden wissen, wobei es dieser $(1969,213)$ «... um Beschreibung 
und Erklärung der ,räumlichen Muster ' und raumbildenden Prozesse der Grundfunktionen menschlicher Existenz» gehe.

Unsere kritische Auseinandersetzung mit dieser Konzeption (die während der letzten vier Jahre auch empirische Arbeiten und das persönliche Gespräch mit den Verfassern einschloß) beschränkt sich im folgenden auf diejenigen Stellen, die für unser System Lebensraum von direkter Bedeutung sind.

Vorerst gilt es festzuhalten, daß die verwendeten Begriffe weder in der ersten Fassung der Konzeption (1969) noch in der modifizierten Kurzfassung von 1973 präzis definiert sind. Dies ist um so gravierender, wenn es sich dabei um Grundbegriffe wie den Begriff der Funktion handelt, auf denen schließlich die ganze Arbeit aufgebaut ist.

Eine genauere Fassung des Funktionsbegriffes als "Aufgabe, Aktivität und Daseinsäußerung» (1969, $208 ; 1973$, 1) hielten die Verfasser wohl deshalb nicht für nötig, weil sie die Interdependenz von Daseinsgrundbedürfnissen und Daseinsgrundfunktionen im (oben kritisierten) Sinne des Funktionalismus auffassen. Auch BIRKENHAUER $(1974,500)$ ist die Diskrepanz zwischen Definition und Bedeutung der Daseinsgrundfunktionen aufgefallen: «... sie sind im mathematischlogischen Sinne nicht ,Funktionen' von etwas, sondern stellen Ansprüche, für die dann erst in der Befriedigung der Ansprüche bestimmte Funktionsebenen und Funktionsträger gefunden werden». Allerdings ist der Fehler nicht in einer falschen Bezeichnung wie BIRKENHAUER meint - sondern in der mangelnden theoretischen Durchdringung des Konzeptes zu suchen.

Ferner ist unerklärlich, daß für die «verorteten Einrichtungen» der Daseinsfunktionen kein neuer Begriff geschaffen wurde, zumal die Geographie «... deren regional differenzierte ,Muster' $\mathrm{zu}$ registrieren und wissenschaftlich zu erklären» $(1969,209)$ habe. Mit einem neuen Begriff hätten Unklarheiten im Argumentationsablauf vermieden werden können.

Der letzte Einwand betrifft die Frage nach den raumrelevanten Gruppen. Wir gehen insofern mit den Verfassern einig, als stets «... eine problembezogene Gruppenbestimmung durchzuführen» $(1969,211)$ ist. Aber «problembezogen» sollte doch heißen: bezogen auf die Kräfte, die raumverändernde Prozesse bewirken. Denn die Geographie soll nicht nur herauszufinden versuchen, wie der Raum verändert wird (Pro- zesse), sondern vor allem auch warum (Dynamik). Mit den «Lebensformgruppen» der Verfasser werden nur diejenigen Gruppen erfaßt, die auf eine (mehr oder weniger) vorgegebene Raumsituation reagieren. Denn die räumlichen Einrichtungen, die sie zur Befriedigung ihrer Daseinsbedürfnisse brauchen, können sie (zumindest in städtischen Räumen) kaum je selbst schaffen. Die Lebensformgruppen sind also darauf angewiesen, daß andere, nämlich die agierenden Gruppen diese Aufgabe übernehmen. Eine Untersuchung, die Prozesse und Dynamik erfassen will, muß also beide Gruppen berücksichtigen.

Wir gehen mit den Verfassern einig, daß wir mehr denn je eine auch für die Praxis leistungsfähige Geographie benötigen. Dazu dürfte aber eine Prognose künftiger Strukturen «aus der Kenntnis der Entwicklungsrichtungen gegenwärtiger Prozesse» - wie sie die «Münchner» sehen - kaum genügen; denn zur Verbesserung dieser Strukturen braucht es lenkende Eingriffe. Solche Eingriffe sind aber nur möglich, wenn die tatsächlichen Ursachen der Prozesse (die Dynamik) erkannt werden. In diesem Sinne ist das richtungsweisende «Münchnen» Konzept zu korrigieren.

\section{Unser Konzept}

Das Konzept der Grunddaseinsfunktionen eignet sich für die Konstruktion des Systems Lebensraum. Weshalb gerade in die gewählten Daseinsbereiche aufgeteilt wurde, läßt sich aus den menschlichen Bedürfnissen nicht ableiten, da diese entsprechend gegliedert vorhanden sein müßten.

An dieser Stelle soll einem Mißverständnis vorgebeugt werden: Grunddaseinsfunktionen werden bei uns nur als analytische Kategorien gebraucht. Sie können nicht als Anleitungen zur Konstruktion von Siedlungen dienen. Daß gerade die Daseinsbereiche als Kategorien gewählt werden, kann pragmatisch begründet werden, was für die empirische Untersuchung ausreichend ist:

1. Durch den engen Zusammenhang von organisatorischer und räumlicher Arbeitsteilung hat sich die organisatorische Aufsplitterung auf den Raum übertragen. In der Siedlung sind somit tatsächlich Einheiten entstanden, die mit den Grunddaseinsfunktionen verglichen werden können. Solche Einheiten werden in der Folge durch Zonenpläne festgelegt, geplant und gebaut. 
2. Diese Bereiche sind als einsichtiges Konzept in der Literatur verbreitet, weil durch sie impliziert wird, sie könnten die für das Dasein der Menschen notwendigen Einheiten bezeichnen.

\section{Allgemeine Begriffe}

Die Begriffe Fläche, Standort, Nutzung, Funktion und Funktionsstelle sind nicht nur für das System Lebensraum von Bedeutung. Deshalb werden sie zuerst allgemein definiert.

Die Fläche bezeichnet als räumliche Grundeinheit einen beliebigen Ausschnitt des zweidimensionalen Raumes. Die Größe der Fläche bewegt sich zwischen 10 und $200000 \mathrm{~m}^{2}$, was der städtischen Parzellengröße entspricht.

Eine Fläche in einer definierten Lage ist ein Standort. Dabei kann die Lage als absolute Lage, d.h. durch das i, j-Koordinatensystem festgelegt, oder als relative Lage, d.h. durch die umgebenden Standorte festgelegt, definiert sein.

Die Art des Gebrauchs einer Fläche oder einer Einrichtung heißt Nutzung.

Kategorien von Tätigkeiten, die auf einen bestimmten Systemzusammenhang hin zweckbezogen sind, heiBen Funktionen. Sie gehören dem sozialen Bereich an. Kategorien von räumlichen Einrichtungen heißen Funktionsstellen, wenn sie Funktionen zugeordnet werden können. Sie gehören dem räumlichen Bereich an. Funktion und Funktionsstelle haben beide den gleichen kategorialen Rahmen und sind auf den gleichen Zweck hin bezogen, wobei Funktion Tätigkeiten, Funktionsstelle räumliche Einrichtungen kategorisiert. Tätigkeiten und räumliche Einrichtungen sind außerhalb der Funktion/Funktionsstelle nicht näher bestimmt, erst durch die Kategorisierung werden sie zweckbe- zogen. Die Funktionsstelle bietet die räumlichen Einrichtungen an einem Standort an, die für die Realisierung der Funktion notwendig sind.

Die beiden Bereiche Raum und Gesellschaft treffen in den Begriffen Funktion und Funktionsstelle aufeinander. Dadurch ist das Postulat der Geographie, die Wirkung zwischen Raum und Gesellschaft zu bestimmen, in diesem Rahmen begrifflich gefaßt worden.

\section{Daseinsgrundfunktion/Daseinsgrundfunktionsstelle}

Funktionen sind auf einen bestimmten Systemzusammenhang hin zweckbezogen. Dieser kann beschrieben werden als System der Konsumtion oder der Versorgung im weiteren Sinn. Solche Funktionen nennen wir Daseinsgrundfunktionen (DGF). Da der Umfang und die Vielfalt der Konsumtion vom gesellschaftlichen Entwicklungsstand abhängig ist, wird der Begriff dem jeweiligen Zustand anzupassen sein.

Allgemein werden durch die DGF die Bedürfnisse des individuellen Lebens oder der individuellen Existenz befriedigt. Bedürfniskategorien, von denen Funktionen abgeleitet werden können, sind nicht definierbar, da Einzelbedürfnisse miteinander so stark verflochten sind, daß durch die Herauslösung eines Bedürfnisses dessen Gehalt verloren ginge. Es ist aber sicherlich berechtigt, hinter jeder DGF ein Bedürfnisbündel anzunehmen, das diese bestimmt, ohne jedoch die einzelnen Bedürfnisse zu benennen. Als sprachliche Erleichterung führen wir für den beschriebenen Sachverhalt den Begriff der Daseinsgrundbedürfnisse (DGB) ein.

Die räumlichen Einrichtungen der DGF heißen entsprechend Daseinsgrundfunktionsstellen (DGS).

Die Beziehungen zwischen den Begriffen lassen sich wie folgt darstellen:

\begin{tabular}{|c|c|c|c|c|c|}
\hline \multicolumn{3}{|l|}{ Sozialer Bereich } & \multicolumn{3}{|c|}{ Räumlicher Bereich } \\
\hline $\begin{array}{l}\text { Konsumtive } \\
\text { Bedürfnisse (DGB) }\end{array}$ & Tätigkeiten & DGF & DGS & Räumliche Einrichtungen & Raum \\
\hline Soziale Bezugseinh & Haushaltung & & & Räumliche Bezugs & tandort \\
\hline
\end{tabular}

Wir übernehmen die Kategorien oder DGF, die in der Literatur gebraucht werden (MALZ 1974, 122; vgl. auch PARTZSCH, RUPPERT/SCHAFFER). Sie werden nachste- hend mit den entsprechenden Tätigkeiten, DGS und räumlichen Einrichtungen aufgeführt: 


\begin{tabular}{|c|c|c|c|}
\hline Tätigkeiten & DGF & DGS & Räumliche Einrichtung \\
\hline Erwerben & Arbeitsfunktion & Arbeitsstelle* & $\begin{array}{l}\text { Werkstatt, Fabrik, } \\
\text { Laden, Büro }\end{array}$ \\
\hline Schlafen, Kochen & Wohnfunktion & Wohnstelle* & Wohnung, Haus \\
\hline Regenerieren, Unterhalten & Erholungsfunktion & Erholungsstelle* & Park, Kino \\
\hline Erarbeiten von Wissen und Erkenntnis & Bildungsfunktion & Bildungsstelle* & Schulhaus, Universität \\
\hline Versorgen i.e.S. & Versorgungsfunktion & Versorgungsstelle* & Laden, Praxis \\
\hline
\end{tabular}

* Zur sprachlichen Vereinfachung ist bei den DGS «Funktion» weggelassen worden.

Da zwischen den einzelnen DGS räumliche Distanzen zu überwinden sind, ist ein Verkehrsnetz notwendig, das die DGS untereinander verbindet. Analog zu diesen führen wir die Begriffe Verkehrsfunktion und Verkehrsstelle ein. An die Verkehrsstelle ist die unverbindliche Kommunikation gebunden. Doch durch die zunehmende Motorisierung ist an diesen Stellen das Kommunizieren immer stärker eingeschränkt worden. Es ist deshalb fraglich, ob das unverbindliche Kommunizieren als eigentliche Kategorie ausgeschieden werden soll.

\section{Lebensraum, Aktionsraum}

Mit den DGS haben wir die Elemente eingeführt, die uns die Konstruktion des Systems Lebensraum erlauben. Dieses System besteht folglich aus den DGS als Elementen und den dazwischenliegenden räumlichen Distanzen als Relationen.

Das System Lebensraum kann auf zwei Arten erfaßt werden:

1. Es können integral alle räumlichen Einrichtungen einer Raumeinheit aufgenommen und kategorisiert werden.

2. Es können zuerst die Aktionsräume aller Haushaltungen erfragt werden. Der Lebensraum ist dann die Vereinigungsmenge der DGS aller Aktionsräume. Diese sind definiert als Systeme, deren Elemente die DGS sind, die von den Mitgliedern der Haushaltungen regelmäßig aufgesucht werden.

Das System Lebensraum ist als statisches System, als Momentaufnahme aufzufassen, das hinsichtlich der räumlichen Verteilung der DGS (sie können verstreut oder konzentriert vorhanden sein) und hinsichtlich der Relationen interpretiert wird. Bei einem «idealen» städtischen System befinden sich möglichst alle DGS in kontrollierter Mischung und die Relationen überschreiten ein gewisses $\mathrm{Maß}$ nicht, welches noch zu erarbeiten ist (vgl. etwa die Größenangaben in WIEGAND 1973, 207-212). Folglich kann ein Stadtsystem von optimaler Größe gefordert werden, das bei volkswirtschaftlich tragbarer Konzentration eine möglichst gute Durchmischung bietet. Auch diese Größe ist noch herauszuarbeiten.

\section{Prozesse}

Unter einem Proze $\beta$ verstehen wir die Veränderung eines Systems in der Zeit. Die Veränderungen spielen sich innerhalb bestimmter Gesetzmäßigkeiten ab: Wenn z. B. ein Element des Systems durch ein anderes ersetzt wird, hat dies wiederum Auswirkungen auf die übrigen Elemente. So wird im System Lebensraum jede Auswechslung von DGS das gesamte System beeinflussen, indem eine Anpassung der übrigen DGS an die neue Situation erfolgt.

Wir meinen im Verdrängungsprozeß eine gesetzmäßige Veränderung der Elemente und Beziehungen des Systems Lebensraum (zumindest für Städte in unserem Wirtschaftssystem) festgestellt zu haben. Mit Verdrängungsprozeß bezeichnen wir die Verdrängung einzelner DGS durch andere DGS. Diese äußert sich in einem Funktionswandel, der dadurch charakterisiert ist, da $ß$ sich an bestimmten Standorten gleiche DGS konzentrieren. Ein polyfunktionaler Raum wird dadurch immer mehr zum monofunktionalen; er wird also funktional entmischt. Am deutlichstèn sichtbar wird dieser Verdrängungsproze $B$ an den vielen StraBenneubauten oder -verbreiterungen, an den Umnutzungen von Wohnraum und am flächenhaften Wachstum. 
Abb. 1 Erkenntnisvorgang

Abb. 1a zeigt den Erkenntnisvorgang mit Hilfe der Systemtheorie in schematischem Ablauf.

Abb. $1 b$ verdeutlicht, daß empirisches Relativ und reales System Teilmengen der totalen Realität sind.

\section{Abb. 2 Hierarchie der Systeme}

Das urbane Metasystem umfaßt zwei Systeme mit je zwei Subsystemen, wobei die Subsysteme als Elemente der Systeme und die Systeme als Elemente des Metasystems aufgefaßt werden können.
Abb. 3 Modell DYN

Das Modell DYN zeigt die Beziehungen der Elemente des DYN-Systems (N, V, S) sowie das Verhalten des DYN-Systems zum System Lebensraum.
$\mathrm{N} \quad=$ Stellennutzer
$\mathrm{V}=$ Stellenverfüger
$\mathrm{S}=$ Stellenschaffer
DGS $=$ Daseinsgrundfunktionsstelle
RS = Renditestelle

(Erläuterung der Zyklen im Text)

(Graphische Bearbeitung: Bruno Baur) la

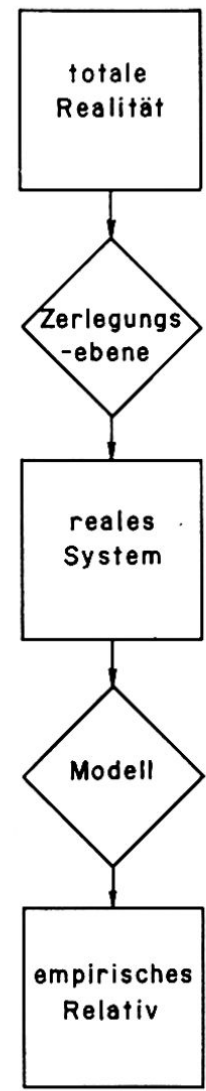

$1 b$

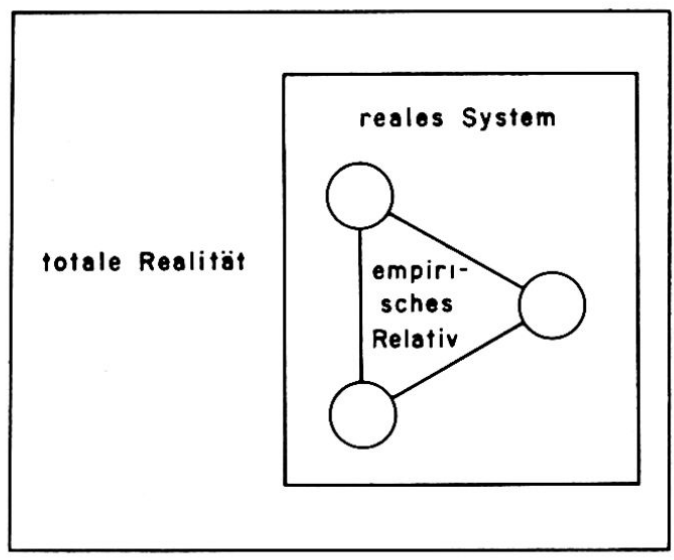

2

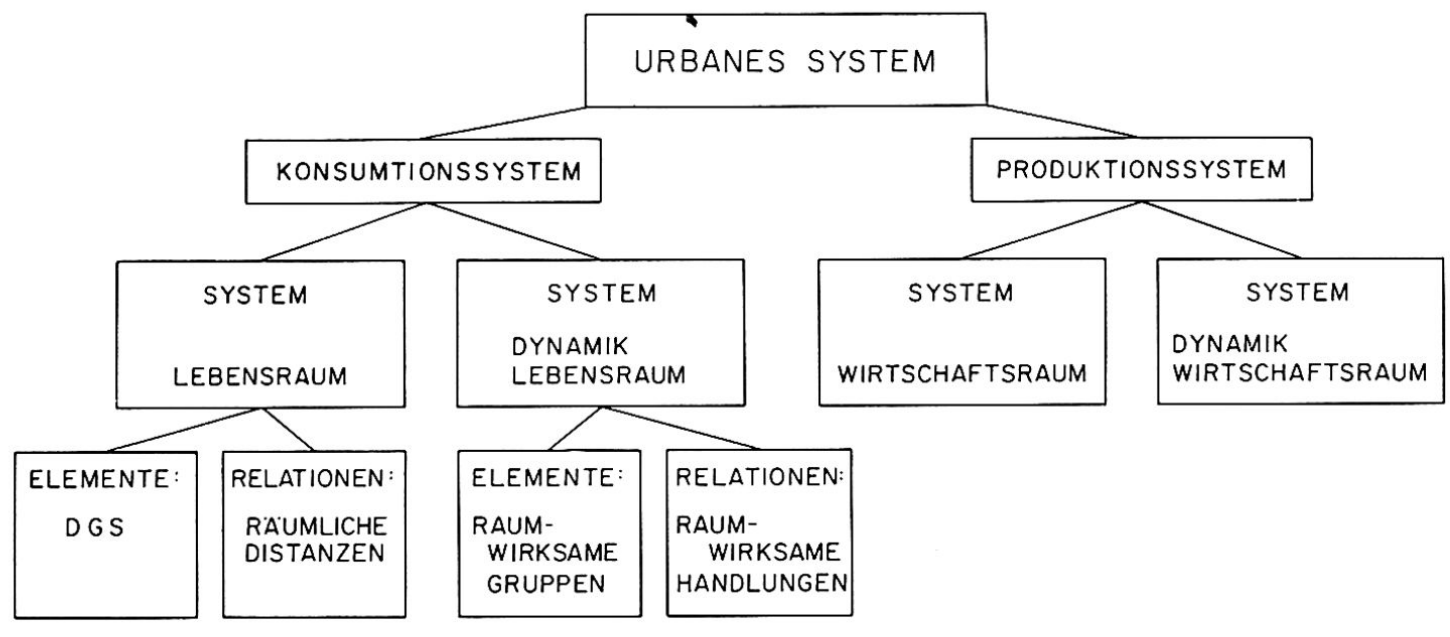

\section{System Dynamik}

3

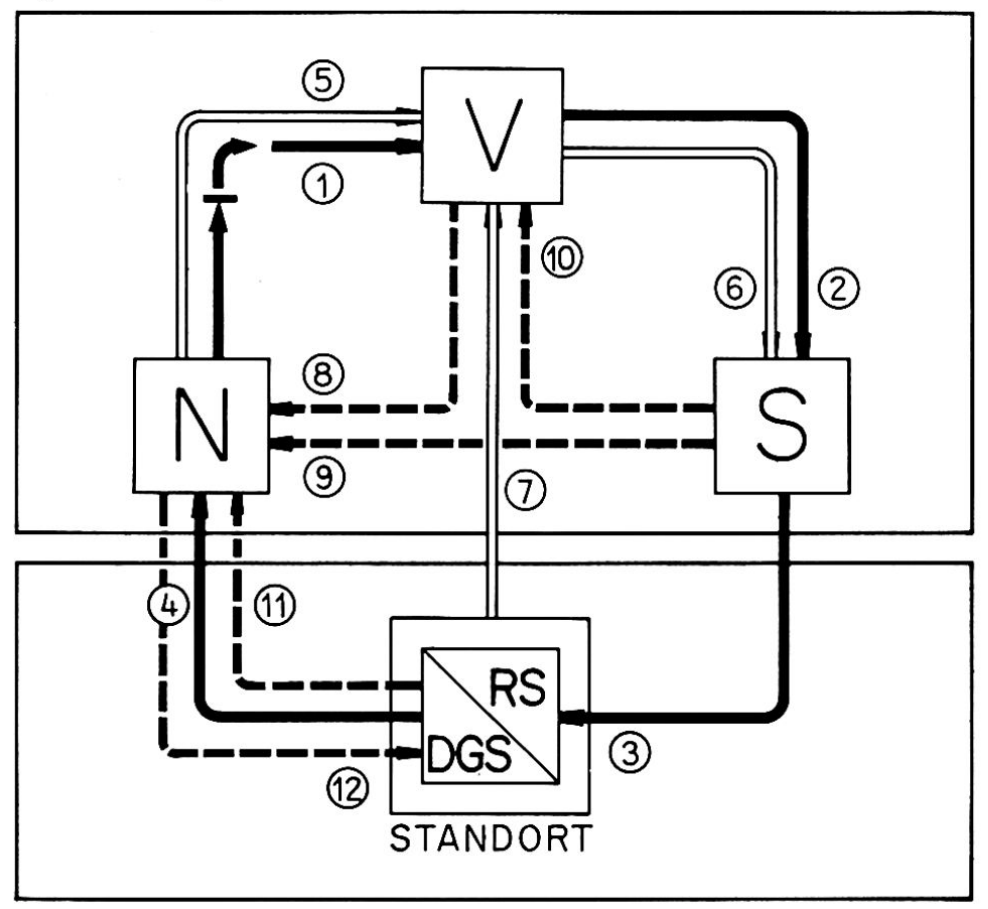

System Lebensraum

DGF-Zyklus 
Der Verdrängungsproze $B$ manifestiert sich somit im Raum als Funktionsstellen- und Morphologiewandel, wobei sich der Wandel der DGS noch stärker auf den Lebensraum auswirkt. Nach einem Wechsel von DGS erfolgt allerdings eine Änderung der Morphologie «... oftmals in erheblichem zeitlichem Abstand hinter dem schnelleren funktionalen Wandel», wie auch HOFMEISTER $(1969,36)$ betont. Stellen, die beinahe gleiche räumliche Ansprüche haben, können einander ablösen, ohne daß große Veränderungen vorgenommen werden müssen. Sind die Ansprüche jedoch verschieden, so ist nach dem Wechsel eine Anpassung des Objektes zu erwarten. Eine Veränderung (Umbau, Neubau) kann aber nur dann erfolgen, wenn bisherige Investitionsleistungen nicht zu hoch sind.

RUPPERT und SCHAFFER (1973, 3 f.) bezeichnen die beharrende Tendenz der Bausubstanz in Anlehnung an DE VRIES REILINGH (1968, «Konsistenz») als Persistenzprinzip. Nach ihrer Auffassung schränken Investitionen «... - orientiert an wirtschaftlichen Rentabilitäts- bzw. gesellschaftlichen Normvorstellungen - die Reaktionsund Aktionsmöglichkeiten der Menschen beträchtlich ein).

\section{Morphologie}

Mit Morphologie der Stadt meinen wir ihre sichtbaren Erscheinungsformen, «d.h. das Straßennetz einschließlich der Plätze, die Bausubstanz und die Freiflächen» (HOFMEISTER 1969, 36).

Für unser Konzept ist die Morphologie nur von Interesse, weil sie von den Entscheidungs- und Handlungsträgern im System Lebensraum als wichtiger Teil der realen Umwelt wahrgenommen wird. Um herauszufinden, wie weit die wahrgenommene Morphologie mit der realen Morphologie übereinstimmt, müssen wir diese miteinander vergleichen. Damit der Vergleich möglich wird, ist vorerst die reale Morphologie zu beschreiben.

Die Ausgestaltung von Straßen- und Platzanlagen sowie die Freiflächen, d.h. die Öffentlichkeitsbereiche, werden im Vordergrund stehen. Aber auch AufrißKriterien werden zu berücksichtigen sein. Solche Untersuchungen wurden von einem der beiden Autoren in Anlehnung an BOBEK und LICHTENBERGER (1966) bereits durchgeführt.

\section{Umweltwahrnehmung}

Die Umwelt wahrnehmen heißt im Bewußtsein Ausschnitte aus der komplexen Realität des Raumes (die auf den Menschen einwirkt) registrieren. Solche Ausschnitte werden aufgrund des individuellen Erfahrungsschatzes wahrgenommen, gedeutet und bewertet (zum Vorgang der Umweltwahrnehmung vgl. HARD 1973, 200-212; mit weiterführenden Literaturangaben).

So kann beispielsweise ein in Wirklichkeit (d.h. in $\mathrm{km}$ gemessen) langer Einkaufsweg als kurz empfunden werden, da er abwechslungsreich gestaltet ist. Die Umwelt wird also nur bedingt so wahrgenommen, wie sie real vorhanden ist.

Andererseits können auch Reaktionen auf real vorhandene Umweltfaktoren erfolgen, ohne daß diese bewußt wahrgenommen werden. So ist beispielsweise jemand aggressiv, weil Verkehrslärm seine Nachtruhe stört, wobei er dies aber vielleicht auf das schlechte Wetter zurückführt.

Die beiden Beispiele illustrieren, warum die Umweltwahrnehmung als Korrekturfakłor ins System Lebensraum einzubeziehen ist: Einerseits kann die individuell oder gruppenspezifisch verschiedene Wahrnehmung des Lebensraumes Entscheidungen und Handlungen unterschiedlich beeinflussen (z. B. dadurch, daß ein negativ konzipierter Lebensraum positiv bewertet wird). Andererseits ist die Umweltwahrnehmung bei Richtwerten für die räumliche Verteilung der DGS (Größe, Mischungsverhältnis) zu berücksichtigen. Ferner spielt sie für die Morphologie eine entscheidende Rolle; denn mit der Errichtung von angeblich «schönen» Gebäuden und Anlagen ist die (sichtbare) Umwelt noch nicht in Ordnung: Es kommt darauf an, wie diese bewertet wird; d. h. ob Bezugspunkte als Möglichkeit zur Identifikation tatsächlich vorhanden sind.

\section{Das System Dynamik (DYN)}

\section{Einleitung}

Prozesse kennzeichnen die Veränderung des Systems Lebensraum in der Zeit. Die Dynamik ist das System der raumwirksamen Kräfte, zeigt also die Ursachen der Prozesse auf.

Im urbanen System gehen die raumwirksamen Kräfte immer von Gruppen oder Individuen aus. Falls es Kräfte gibt, die vom Raum ausgehen und auf den Raum wirken, so spielen sie nur eine untergeordnete Rolle. Hingegen können Entscheidungen aufgrund 
einer Bewertung des räumlichen Systems getroffen werden; der Raum wirkt somit mittelbar auf sich selbst ein.

Gruppen sind raumwirksam, wenn von ihnen Kräfte ausgehen, die auf das Raumsystem eine Wirkung ausüben. Im System Lebensraum können die Funktionsträger, also Haushaltungen, als Gruppen bezeichnet werden. Es stellt sich nun die Frage, ob diese Haushaltsgruppen raumwirksam sind. In beschränktem Umfang können sie direkt auf die DGS einwirken, meistens aber werden nur Reaktionen auf die DGS hervorgerufen. Diese Gruppen bezeichnen wir deshalb als reagierende, betroffene Gruppen.

Die Tätigkeiten und räumlichen Einrichtungen können auch auf einen anderen Systemzusammenhang hin zweckbezogen sein, nämlich darauf, Rendite zu erzielen. Tätigkeiten haben dann Renditefunktionen $(R F)$, räumliche Einrichtungen sind Renditestellen $(R S)$. Die Träger der Renditefunktion nennen wir agierende Gruppen. Diese sind in großem Umfang raumwirksam. Mit Rendite ist nur die in unserem Wirtschaftssystem wichtigste Motivation aufgezeigt worden. In Einzelfällen können aber andere Motivationen zugrunde liegen (vgl. auch die Ausnahmefälle im Kapitel «Prozeßverlauf»).

Die agierenden und reagierenden Gruppen sind im seltensten Fall miteinander identisch. Daraus entsteht eine große Diskrepanz, die für die meisten Systemmängel verantwortlich gemacht werden kann. Auf diesen beiden Gruppen bauen wir das Modell DYN auf.

\section{Modell DYN}

Elemente des DYN-Systems sind die raumwirksamen Gruppen, Relationen sind raumwirksame Handlungen dieser Gruppen. Als raumwirksame Gruppen unterscheiden wir die Stellennutzer $(N)$, die Stellenverfüger (V) und die Stellenschaffer (S); ferner wird das Verhalten des Systems DYN zum System Lebensraum miteinbezogen (Abb. 3).

\section{Die Stellennutzer $(N)$}

Die Gruppe der Stellennutzer des Systems DYN ist identisch mit der Gruppe der DGF-Träger des Systems Lebensraum. Die Stellennutzer haben nur indirekten und bescheidenen Einfluß auf die DGS, sofern sie nicht mit den Stellenverfügern oder Stellenschaffern identisch sind.

\section{Die Stellenverfüger $(V)$}

Die Gruppe der Stellenverfüger veranlaßt die Schaffung von Stellen, die zugleich DGS und RS sein müssen. Die Stellenverfüger sind meistens Standorteigentümer (Grundstückeigentümer) und/oder verfügen über das zur Errichtung der Stellen notwendige Kapital. Der Anstoß zur Schaffung von Stellen, der von den Stellennutzern ausgeht, ist meistens nicht faßbar. Es werden also in den seltensten Fällen direkte Raumbedürfnisse der Stellennutzer befriedigt.

\section{Die Stellenschaffer (S)}

Die Gruppe der Stellenschaffer errichtet auf Veranlassung der Stellenverfüger die Stellen. Die Stellenschaffer sind an den Stellen nur insofern interessiert, als sie Bausubstanz herstellen, die Rendite verspricht.

Folgende Identitäten können auftreten:
$\mathrm{N}=\mathrm{V}=\mathrm{S}$
$\mathrm{N}=\mathrm{V}$
$\mathrm{N}=\mathrm{S}$
$\mathrm{V}=\mathrm{S}$

Sind die Stellenverfüger und die Stellenschaffer identisch, so ist ihr Einfluß auf die Stelle groß. Noch größer ist er, wenn alle drei zusammenfallen. Nicht viel weniger bedeutend ist der Einfluß, wenn Stellenverfüger und Stellennutzer identisch sind. Dabei gilt es allerdings zu beachten, daß einzelne Wohlhabende sich zwar verschiedene DGS nach ihrem Belieben einrichten, aber niemals das ganze System Lebensraum direkt verändern können.

Mit den raumwirksamen Gruppen als Elementen und ihren raumwirksamen Handlungen als Relationen kann das Modell der Abb. 3 entwickelt werden. Es ist wie folgt zu interpretieren:

\section{DGF-Zyklus}

1 Nutzer $(\mathrm{N})$ benötigen zur Befriedigung von Daseinsgrundbedürfnissen (DGB) räumliche Einrichtungen. Mit der Nachfrage nach Daseinsgrundfunktionsstellen (DGS) wird dieser Umstand dem Verfüger (V) zur Kenntnis gebracht. In der Regel handelt es sich dabei um eine allgemeine, nicht gruppenbezogene Nachfrage.

2 Der Verfüger veranlaßt den Schaffer (S), entweder auf seinem Standort (Boden) oder mit seinem Kapital Renditestellen (RS) bzw. DGS zu errichten.

3 Der Schaffer baut ensprechende RS/DGS.

4 Die DGS werden den Nutzern zur Verfügung gestellt und von diesen gebraucht. 
RF-Zyklus

5 Die Nutzer entschädigen den Verfüger (z. B. in Form von Mieten).

6 Der Verfüger entschädigt den Schaffer.

7 DGS sind für den Verfüger Kapitalanlagen.

Korrektur-Zyklus

8 Der Verfüger beeinflußt den Nutzer zur Korrektur seiner Bedürfnisse.

9 Der Schaffer beeinflußt den Nutzer zur Korrektur seiner Bedürfnisse.

10 Der Schaffer übt Einfluß auf den Verfüger aus und bewegt diesen dazu, den Bau von Stellen zu veranlassen.

11 Die DGs wirken auf das Verhalten des Nutzers (räumliche Kräfte).

12 Der Nutzer nimmt Änderungen an der DGS vor.

\section{Prozeßauslösung}

Ein Prozeß im System Lebensraum wird durch einen Prozeß im DYN-System ausgelöst; denn Handlungen, die räumliche Veränderungen bewirken (also raumwirksam sind) beruhen auf Entscheidungen, und diesen liegt wiederum eine Raumbewertung zugrunde (vgl. dazu etwa CARTERs Ausführungen über «aspatial-/spatial aspects» 1973, $10 \mathrm{ff}$.).

Bei der Raumbewertung wird zunächst der Standort beurteilt. Je nach dem, welche Bewertungskriterien im Vordergrund stehen, bringt ein Standort Vorteile. In der folgenden Liste sind sieben mögliche Standortvorteile abgegrenzt, die einzeln oder beliebig kombiniert auftreten können:

1. Verkehrslage: Lage an einem leistungsfähigen Verkehrsträger

2. Repräsentationslage: Lage, die dem Standort Prestige verspricht

3. Horizontale Nachbarschaftslage: die umgebenden Betriebe gehören der gleichen Branche an

4. Vertikale Nachbarschaftslage: die umgebenden Betriebe gehören nicht der gleichen Branche an, sind aber für diese von Bedeutung

5. Geländelage: Lage im Gelände, das für den Standort die notwendigen Voraussetzungen mitbringt

6. Erschließungsgrad

7. Bauzonenvorschriften.

Ein Standort ist meistens für verschiedene DGS günstig. Dem marktwirtschaftlichen Gesetz von Angebot und Nachfrage gemäß kommt es dann zum Konkurrenz- kampf, wenn mehrere Stellenverfüger Anspruch auf den Standort erheben; denn beim Standort handelt es sich ja um Boden, also unvermehrbares Gut in einer bestimmten Lage. Die Nachfrage wiederspiegelt sich denn auch im Muster der Bodenpreise.

Damit der Standort rentiert, muß der Bodenpreis durch die Flächenrendite kompensiert werden. Deshalb wird diejenige DGS den Konkurrenzkampf um den Standort gewinnen, die am Standort pro Flächeneinheit die größte Rendite erzielen kann oder zu erzielen hofft. Entscheidend ist somit die Wertigkeit der DGS.

Wenn DGS gleiche Flächenrendite erzielen, bezeichnen wir sie als gleichwertig. Entsprechend lassen sich höher-, bzw. tieferwertige DGS definieren.

Dieser Umstand läßt eine Hierarchie der DGs, d. h. eine Aufreihung der Stellenbereiche entsprechend ihrer Wertigkeit vermuten. Wir verzichten an dieser Stelle auf eine Hierarchie-Liste, da sie empirisch erhobene Daten voraussetzt.

\section{Prozeßverlauf}

Halten wir nochmals fest: räumliche Prozesse werden durch nicht-räumliche ausgelöst. Im Sinne der Abb. 3 heißt dies, daß das Verhalten des DYN-Systems Reaktionen im System Lebensraum bewirkt, was wiederum Rückwirkungen auf das System DYN zur Folge hat. Dieser Systemzusammenhang ist am einfachsten aus dem Prozeßablauf einer einzelnen DGS zu erkennen:

1. Die DGS $_{1}$ wird genutzt.

2. Infolge von Innovationen
a) verkehrstechnischen Maßnahmen
b) neu erstellten DGS in der Nachbarschaft
c) sonstigen, systemexternen Einwirkungen
fallen dem Standort der DGS ${ }_{1}$ neue Vorteile zu.

3. Raumwirksame Gruppen (des DYN-Systems) bewerten den Standort der $\mathrm{DGS}_{1}$ neu, sofern sie einen Standort mit solchen Vorteilen benötigen.

4. Wenn der Standort als günstig beurteilt wird, steigt die Nachfrage; d. h. eine DGS $_{2}$ oder mehrere $\mathrm{DGS}_{\mathrm{n}}$ erheben Anspruch auf den Standort der DSG $\mathrm{G}_{1}$.

5. Nun setzt der Konkurrenzkampf um den Standort der $\mathrm{DGS}_{1}$ ein. Er wird von derjenigen $\mathrm{DGS}_{1, \mathrm{n}}$ gewonnen, die pro Flächeneinheit die größte Rendite erzielt, also die höchste Wertigkeit aufweist (Prozeß im DYN-System).

In Ausnahmefällen ist aber nicht die Flächenrendite entscheidend: 
a) eine individuelle Resistenz, die potentielle Gewinne ausschlägt und Mehrkosten in Kauf nimmt, wird in einem gewissen Rahmen der Flächenrendite widerstehen können;

b) sofern aus Repräsentationsgründen ein durch die DGS entstehender Verlust einkalkuliert wird, spielt die Flächenrendite keine Rolle;

c) falls der Staat oder eine öffentlich-rechtliche Organisation die Verfügungsgewalt über den Standort hat, spielt die Flächenrendite keine Rolle.

6. Wenn die neue $\mathrm{DGS}_{\mathrm{n}}$ höherwertig ist, wird

a) die alte $\mathrm{DGS}_{1}$ verdrängt und die neue $\mathrm{DGS}_{\mathrm{n}}$ auf dem Standort eingerichtet (Prozeß im LebensraumSystem);

b) der Bodenpreis (Handänderung) steigt, da sich in ihm Standortvorteile bzw. Flächenrendite wiederspiegeln.

7. Die neue $D_{G S}$ wird genutzt.

8. Wenn die verdrängte $\mathrm{DGS}_{1}$ einen neuen Standort sucht, kommt es irgendwo im System zu einer Neubewertung eines Standortes und somit erneut zu einem Prozeßablauf gemäß den Punkten 3 bis 7 .

\section{Prozessuale Stadtdifferenzierung}

Im soeben beschriebenen Prozeßverlauf wird eine DGS durch eine andere ersetzt. Es werden also Elemente im System Lebensraum ausgewechselt; somit verändert sich auch das System. Solche Prozesse spielen sich zwar im gesamten System ab, aber nicht überall

a) in der gleichen Intensität (Prozeß-Phase) und

b) in der gleichen Art (Wertigkeitsstufe).

Deshalb ist das System Lebensraum in Teilsysteme aufzuteilen.

Teilsysteme lassen sich durch Prozeß-Phasen und Wertigkeits-Stufen definieren, in der sie sich zu einem bestimmten Zeitpunkt befinden. Die Stufe wird durch die Wertigkeit der im Teilsystem vorherrschenden DGS bestimmt. Ein Teilsystem durchläuft drei ProzeßPhasen:

\section{Nullphase}

Es werden nur sporadisch DGS ausgewechselt, wobei keine gesetzmäßige Verdrängung (vgl. Kapitel «Prozesse») festgestellt werden kann. Das Teilsystem ist stabil.

\section{Innovationsphase}

Bestimmte DGS werden zwar noch punktuell, aber infolge einer Innovation gesetzmäßig verdrängt. Das Teilsystem wird labil.

\section{3. Übergangsphase}

Die verdrängenden DGS werden vorherrschend. Das Teilsystem ist noch labil, beginnt sich aber zu stabilisieren. Da die Wertigkeit der nun vorherrschenden DGS gestiegen ist, hat sich ein Stufenwechsel vollzogen.

\section{(4. Nullphase}

Das Teilsystem ist auf einer höheren Stufe stabil.) Die zeitliche Dauer der einzelnen Prozeßphasen ist nicht bestimmbar, da sie von verschiedenen, systemexternen Ursachen abhängig ist. Grenzwerte, welche die Phasen gegeneinander absetzen, können erst während der weiteren Operationalisierung festgelegt werden.

Im System Lebensraum treffen wir somit Teilsysteme an, die auf unterschiedlicher Stufe und in unterschiedlicher Phase stehen. Die Stadtregion läßt sich aufgrund dieser Teilsysteme gliedern. Dabei kann generell unterschieden werden, ob es sich um labile oder stabile Teilsysteme handelt.

Mit der Erfassung dieser Teilsysteme wird nicht nur aufgezeigt, wie (Prozesse) und warum (Dynamik) sich der Lebensraum verändert. Auch die traditionellen geographischen Anliegen, nämlich wo (Verortung) und mit welcher Intensität (Differenzierung) sich diese Vorgänge abspielen, werden berücksichtigt. Nur wenn wir alle vier Aspekte kennen, besitzen wir die Voraussetzungen, um Veränderungen des Lebensraumes im Sinne einer nach Bedürfnissen der Bewohner gestalteten Stadt beeinflussen zu können.

\section{Zusammenfassung}

Stadtgeographische Forschung sollte den urbanen Raum in seiner Veränderung erklären. In dieser Richtung wird sie praxis- und gesellschaftsbezogen wirken, indem sie beispielsweise erfolgreiche planerische Eingriffe ermöglicht. Solche Eingriffe sind erst wirkungsvoll, wenn außer den Veränderungen (prozessuale Betrachtungsweise bisheriger Konzepte) auch deren Ursachen (neu: Dynamik) erkannt werden. Im urbanen Raum werden Veränderungen vor allem von sozialen und nur beschränkt von natürlichen Kräften verursacht. Ein stadtgeographischer Ansatz muß deshalb zugleich ein sozialgeographischer Ansatz sein.

$\mathrm{Um} \mathrm{zu}$ fundierten Aussagen zu gelangen, muß der empirischen Arbeit eine theoretische Durchdringung 
des Untersuchungsgegenstandes vorausgehen. Im vorliegenden Artikel wird aufgrund systemtheoretischer Überlegungen ein Stadtmodell entwickelt, das in der Gemeinschaftsdissertation der Autoren empirisch überprüft und mit diesem Artikel zur Diskussion gestellt wird.

Im größeren Systemzusammenhang urbaner Raum steht das System Lebensraum im Vordergrund. Darunter wird der Raum verstanden, der - zur Konsumtion im weitesten Sinne - der urbanen Bevölkerung zur Verfügung steht. Im System Lebensraum ist das Postulat der Geographie, die Wirkung zwischen Raum und Gesellschaft $\mathrm{zu}$ bestimmen, begrifflich (Funktion, Funktionsstelle) gefaßt worden. Der Systemaufbau basiert auf den Daseinsgrundfunktionen, die vom Funktionalismus abgegrenzt werden. Der Verdrängungsproze $B$ einzelner Daseinsgrundfunktionsstellen, so heißen die räumlichen Einrichtungen der Daseinsgrundfunktionen, ist als wichtiger Prozeß erkannt worden.

Im System Dynamik wird ein System der Kräfte entworfen, die diese Prozesse bewirken. Dabei werden zwei raumwirksame Gruppen unterschieden: die erste Gruppe tritt als Nutzer, die zweite Gruppe als Verfüger und Schaffer der Daseinsgrundfunktionsstellen in Erscheinung.

Entscheidungen, die räumliche Veränderungen nachsichziehen, gehen unterschiedliche Raumbewertungen voraus. Diese müssen erkannt und die Veränderungen lokalisiert werden, wenn lenkende Eingriffe erfolgen sollen. Aus dieser Sicht dürfte die aufgezeigte prozessuale Differenzierung der Stadtregion von Interesse sein.

\section{Literatur}

AUTORENGEMEINSCHAFT (1973): Vorschlag für einen neuen Geographielehrplan. Aufschluß. Info. der Geogr. Fachgruppe Basel, H. 2

BIRKENHAUER, J. (1974): Die Daseinsgrundfunktionen und die Frage einer "curricularen Plattform» für das Schulfach Geographie. Geogr. Rundschau 26, 499-503 BOBEK, H. (1927): Grundfragen der Stadtgeographie. Geogr. Anzeiger, 213-224
BOBEK, H. (1948): Stellung und Bedeutung der Sozialgeographie. Erdkunde, 118-125

BOBEK, H. Und LICHTENBERGER, E. (1966): Wien. Bauliche Gestalt und Entwicklung seit der Mitte des 19. Jahrhunderts. Graz/Köln

BURCKHARDT, L. und FÖRDERER, w. (1972): Bauen ein ProzeB. 2. Aufl. Teufen

CARTER, H. (1973): The Study of Urban Geography, 2. Auflage. London

DE VRIES REILINGH, H. (1968): Gedanken über die Konsistenz in der Sozialgeographie. Münchner Stud. zur Sozial- u. Wirtschaftsgeogr. 4, 109-118

FRIEDRICHS, J. (1973): Methoden empirischer Sozialforschung. Rororo Studium 28 (Reihe: Sozialwissenschaft). Reinbek bei Hamburg

FÜGLISTER, H., KÜPFER, D. Und LÖTSCHER, L. (1974): Das Bruderholz als Naherholungsgebiet. Regio Basiliensis $14,375-414$

GALLUSSER, w. (1970): Struktur und Entwicklung ländlicher Räume der Nordwestschweiz. Aktualgeogr. Analyse der Kulturlandschaft im Zeitraum 1955-1968. Basler Beiträge zur Geogr. 11

GALLUSSER, W. (1971): Die Veränderung der Umwelt als aktualgeographisches Problem. Regio Basiliensis 12, 174-182

HAGGET, P. (1965): Locational Analysis in Human Geography. London

HARBORDT, S. (1974): Computersimulation in den Sozialwissenschaften. Band 1: Einführung und Anleitung. Rororo Studium 49 (Reihe Sozialwissenschaft). Reinbek bei Hamburg

HARD, G. (1973): Die Geographie. Eine wissenschaftstheoretische Einführung. Sammlung Göschen 9001. Berlin/New York

HOFMEISTER, B. (1969): Stadtgeographie. Das geogr. Seminar. Braunschweig

ISBARY, G. (1971): Raum und Gesellschaft. Beiträge zur Raumordnung und Raumforschung aus seinem Nachlaß (Bearbeitet von D. PARTZSCH). Veröff. der Akademie für Raumforschung und Landesplanung, Beiträge, 6 . Hannover

KLAUS, G. (1969): Wörterbuch der Kybernetik. Fischer Handbücher 1073/1074. Frankfurt a. M./Hamburg

KRIZ, J. (1973): Statistik in den Sozialwissenschaften. Rororo Studium 29 (Reihe: Sozialwissenschaft). Reinbek bei Hamburg 
LASCHINGER, w. (1974): Eine städtische Luftbildinterpretation: das nördliche Kleinbasel. Regio Basiliensis $14,366-374$

LE CORBUSIER (1943): La Charte d'Athènes. Paris LE CORBUSIER (1962): An die Studenten. Die Charta von Athen. Reinbek bei Hamburg

LENG, G. (1973): Zur «Münchnen» Konzeption der Sozialgeographie. Geogr. Zeitschr. 61, 121-134

LEXIKON ZUR SOZIOLOGIE (1973). Opladen

LÖTSCHER, L. (1971): Studien zur siedlungsräumlichen Dynamik der Gemeinde Birsfelden seit dem 19. Jahrhundert (Unveröffentlichte Lizentiatsarbeit). Basel

LÖTSCHER, L. (1972): Birsfeldens aktuelle Siedlungsphysiognomie. Regio Basiliensis 13, 10-21

LÖTSCHER, L. (1975): Wandel des Lebensraumes. Heimatkunde von Birsfelden

MALZ,F. (1974): Taschenwörterbuch der Umweltplanung. LTw 1614 (Reihe: Sozialwissenschaften). München

MITSCHERLICH, A. (1971): Die Unwirtlichkeit unserer Städte. Ed. Suhrkamp 123. 11. Aufl. Frankfurt a. M.

MONKHOUSE, F. J. (1970): A Dictionary of Geography. Sd. Ed. London

PARTZSCH, D. (1964): Zum Begriff der Funktionsgesellschaft. Mitt. des Dt. Verbandes für Wohnungswesen, Städtebau und Raumplanung. H. 4, 3-10
PARTZSCH, D. (1966): Funktionsgesellschaft. Handwörterbuch der Raumforschung und Raumordnung. Hannover

RUPPERT, K. und SCHAFFER, F. (1969): Zur Konzeption der Sozialgeographie. Geogr. Rundsch. 21, 205-214

RUPPERT, K. und SCHAFFER, F. (1973): Sozialgeographische Aspekte urbanisierter Lebensformen. Abh. der Akad. f. Raumforschung u. Landesplanung 68. Hannover

RUPPERT, K. und SCHAFFER, F. (1974): Zu G. Leng's Kritik an der «Münchnen» Konzeption der Sozialgeographie. Geogr. Zeitschr. 62, 114-118

WIEGAND, J. (1973): Funktionsmischung. Zur Planung gemischter Gebiete als Beitrag zur Zuordnung von Wohn- und Arbeitsstätten. Burckhardt Berichte 1. Basel

PRAKTIKUMSARBEITEN (unveröffentl. Manus.) aus dem Geogr. Inst. der Univ. Basel:

HEIM, V. und SCHMIDLIN, R. (1974): Kleinstädte als Lebensraum (Arbeitsexkursion in die drei Kleinstädte Langenthal, Burgdorf und Herzogenbuchsee)

BROGLE, C., FEHR, S., HEIM, V., JOOS, H., MATTER, W., PELET, P. und WEBER, H. (1974): Städtische Entmischung. Vergleichende Untersuchung eines Stadtbezirks und einer Wohnsiedlung am Stadtrand: Landskron und Sternenfeld 